DIVISION OF THE HUMANITIES AND SOCIAL SCIENCES

CALIFORNIA INSTITUTE OF TECHNOLOGY

PASADENA, CALIFORNIA 91125

THE POSITIVE FOUNDATION OF THE COMMON PRIOR ASSUMPTION

Aviad Heifetz

Tel Aviv University

and California Institute of Technology

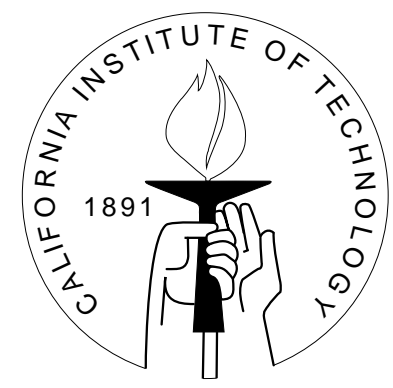

SOCIAL SCIENCE WORKING PAPER 1125

July 2001 


\title{
The Positive Foundation of the Common Prior Assumption
}

\author{
Aviad Heifetz
}

\begin{abstract}
The existence of a common prior is a property of the state space used to model the players' asymmetric information. We show that this property is not just a technical artifact of the model, but that it is immanent to the players' beliefs. To this end, we devise a condition, phrased solely in terms of the players' mutual beliefs about the basic, objective issues of possible uncertainty, which is equivalent to the existence of a common prior.
\end{abstract}

JEL classification numbers: D80, D82, D84

Key words: common prior, asymmetric information 


\title{
THE POSITIVE FOUNDATION OF THE COMMON PRIOR ASSUMPTION
}

\author{
Aviad Heifetz*
}

February 2001

\section{Introduction}

The common prior assumption (Harsanyi 1967-68) is pervasive in most economic models of asymmetric information. It asserts that the beliefs of individuals in different states of the world are the posteriors they form given their private information from a prior which is common to them all. Morris (1995) elaborates upon the possible normative justifications for this assumption.

However, when states of the world are constructed from explicit descriptions of the individuals' hierarchies of mutual beliefs (Mertens and Zamir 1985), it turns out that belief subspaces with a common prior are the exception rather than the rule. In particular, a common prior does not emerge from any natural assumption in this explicit construction of economic types. The positive value of the common priors assumption is therefore at stake: By examining the beliefs of individuals at a given situation (or "state of the world"), can one tell whether they originate from a common prior? If the answer is negative, it means that having a common prior can only be meaningful for the modeler,

*The Division of Humanities and Social Sciences, The California Institute of Technology and The School of Economics, Tel Aviv University, heifetz@post.tau.ac.il 
who observes it as a mere technical regularity across the states in the model, with no economic content. If, on the other hand, the answer is positive, the assumption is testable, and in any specific economic interaction we can check for its validity by eliciting the beliefs of individuals in the situation at hand.

Up till now, the literature offers characterizations of the common prior assumption in terms of the expectations the individuals ascribe to random variables (Morris 1994, Samet 1998a,b, Halpern $1998^{1}$ ). In the way they are presented, it is not clear whether these characterizations are positive in the above sense: To describe a random variable, the individuals have to know the structure the of the state space, which is an abstract construct of the modeler, and not necessarily the form in which the individuals grasp the uncertain environment in which they act. Alternatively, Feinberg (2000) offers a characterization in terms of the individuals' beliefs regarding events in an augmented state space, which incorporates the outcomes of a commonly known randomizing device. Here again, an exogenous construct (the randomizing device) has to be alluded to.

The main contribution we propose here is in showing that when the state space is compact, we have a positive characterization for the existence of a common prior, which requires only to elicit the individuals' mutual beliefs on the fundamentals of the interaction (the basic or "nature" events), like payoffs entailed by the possible actions. In such an enquiry, the questions posed to the individuals can be phrased using expressions in natural language, without referring to any model or abstract structure.

Here is the essence of the argument for the case of two individuals. There is no common prior among the individuals if and only if there is a zero-sum bet that they are both willing to take (as expected value maximizers), and such that this fact is common belief between them (Feinberg 2000). The bet is a continuous random variable. Since the space is compact, it is also common belief between them that their assessed expected gains from the bet are bounded away from zero. This turns to imply that we can uniformly approximate this bet by a simple zero-sum bet, whose finitely many possible outcomes depend on events described by expressions with finitely many words each ${ }^{2}$, and such that the subjectively assessed gains of the new bet are still commonly believed to be positive and bounded away from zero. And though the exact individual assessments of the bet are not commonly known, it turns out that there is a finite set of sufficient justifications to like the bet, such that it is commonly believed that at least one of these justifications obtains for each individual. These justifications are phrased in terms of bounds on the probabilities of the expressions which determine the outcome of the bet. Therefore, this set of justifications can also be described with finitely many words, and common belief that one of them obtains is equivalent to the lack of a common prior.

Section 2 presents the technical preliminaries and definitions. Section 3 contains the statement and proof of the main result. The appendix brings a very short proof to a

\footnotetext{
${ }^{1}$ Halpern 1998 uses a logical syntax which express the individuals' expectations of random variables with finitely many values.

${ }^{2}$ Expressions will be formally defined in the following section.
} 
characterization of common priors in compact spaces due to Feinberg (2000), generalizing a technique of Samet (1998).

\section{Preliminaries}

A (topological) type space is a Hausdorff space $\Omega$, such that every individual $i$ in the set $\{1, \ldots, I\}$ of individuals has a regular Borel probability measure $t_{i}(\omega)$ on $\Omega$ in every state $\omega \in \Omega$. This type mapping $t_{i}$ from $\Omega$ to the space $\Delta(\Omega)$ of regular Borel probability measures is assumed to be continuous, when $\Delta(\Omega)$ is endowed with the topology of weak convergence ${ }^{3}$.

The Borel subsets $\Sigma$ of $\Omega$ are called events. A countable subfield $\Sigma_{0} \subseteq \Sigma$ constitutes the set of basic or natural events. These are events that describe objective circumstances, like "NASDAQ went up X\% today" or "this combination of actions by the individuals will result by those payoffs for them", but not any assertion that involves the beliefs or knowledge of the individuals.

For every event $E$ and a rational number $p \in[0,1]$,

$$
B_{i}^{p}(E)=\left\{\omega \in \Omega: t_{i}(\omega)(E) \geq p\right\}
$$

is the event "individual $i$ assigns probability at least $p$ to the event $E$ " 4 ;

$$
\neg E=\Omega \backslash E,
$$

the complement of $E$, is the event "not $E$ ". For two events $E$ and $F$,

$$
E \cap F
$$

is the event " $E$ and $F$ ".

The field $\mathcal{E}$ of expressions is the one generated from the basic events $\Sigma_{0}$ by using consecutively $\neg, \cap$ and $B_{i}^{p}$. Notice that the collection of expressions is countable, and

\footnotetext{
${ }^{3}$ This topology is generated by the sub-basis of sets of the form
}

$$
\{\mu \in \Delta(\Omega): \mu(O)>r\}
$$

where $O \subseteq \Omega$ is open and $r \in R$ (see e.g Billingsley 1968, appendix III). When $\Omega$ is Normal (and in particular compact and/or metric), this topology coincides with the weak-* topology - the weakest topology for which the mapping

$$
f \rightarrow \int_{\Omega} f \mathrm{~d} \mu
$$

is continuous for every contiunuous real-valued function $f$ on $\Omega$.

${ }^{4}$ The continuity of $t_{i}$ guarantees that $B_{i}^{p}(E)$ is indeed an event in $\Sigma$. 
thus can be enumerated in a sequence. We say that the expression $E$ holds in a state $\omega \in \Omega$ when $\omega \in E$. The set of expressions $D(\omega)$ that hold in the state $\omega$ is called the description of $\omega$. Denote by

$$
\mathcal{D}=\{D(\omega): \omega \in \Omega\}
$$

the set of descriptions of the states in $\Omega$.

We say that an expression $E$ is common belief at $\omega$ if $B_{i_{1}}^{1}\left(B_{i_{2}}^{1}\left(\ldots B_{i_{k}}^{1} E\right)\right)$ holds in $\omega$ for every sequence $i_{1}, i_{2}, \ldots, i_{k} \in\{1, \ldots, I\}$.

We will assume that the set $\mathcal{O}$ of open expressions is a basis for the topology of $\Omega$, and therefore that the field $\mathcal{E}$ of expressions generates the Borel $\sigma$-field $\Sigma$ of events. In particular, different descriptions hold in different states. ${ }^{5}$

Expressions and descriptions are objects for a positive enquiry. At a given situation, one can check whether certain expressions hold without having to refer explicitly to other situations, as captured formally by other states of the model. This is important, because the states of the model are abstract constructs defined by the modeler, but this is not necessarily the way the modeled individuals capture the situation. Thus, to answer a question whether she would take a certain gamble whose outcome depends on the states of $\Omega$, the individual has to understand what $\Omega$ is. In contrast, every expression can be phrased with finitely many words and without any reference to $\Omega$.

A probability measure $P_{i} \in \Delta(\Omega)$ is a prior for individual $i \in\{1, \ldots, I\}$ if for every event $E \in \Sigma$

$$
P_{i}(E)=\int_{\Omega} t_{i}(\omega)(E) \mathrm{d} P_{i}(\omega)
$$

The probability measure $P \in \Delta(\Omega)$ is a common prior if it is a prior for each of the individuals $i \in\{1, \ldots, I\}$.

In the analysis below, we will assume that $\Omega$ is not the union of proper beliefsubspaces, i.e. that it is not the case that for some event $E \subsetneq \Omega$ we have both

$$
\begin{gathered}
t_{i}(\omega)(E)=1 \quad \forall \omega \in E, i \in\{1, \ldots, I\} \\
t_{i}(\omega)(\neg E)=1 \quad \forall \omega \in \neg E, i \in\{1, \ldots, I\}
\end{gathered}
$$

(because if that were the case, we could elaborate upon the existence of a common prior separately in $E$ and $\neg E$ ).

\footnotetext{
${ }^{5}$ This means that $\Omega$ is non-redundant, in the sense that different states indeed represent different situations. For example, if $\Omega$ has two states, it is not the case that every basic event $E_{0}$ coincides either with $\Omega$ or with the empty set, because otherwise the same description would obtain in both states of $\Omega$ : Even if the individuals had different beliefs across the two states their mutual beliefs on the basic events would always coincide. In other words, in such a redundant space the different types of an individual across the two states do not differ in any substantial way.
} 


\section{A Positive Characterization of Common Priors}

The main result of this work is the characterization of common priors in terms of expressions and descriptions when the type space is compact. We first phrase and prove it for the case of two individuals, and generalize the result to an arbitrary finite number of players.

For the positive characterization we rely on the following proposition due to Feinberg (2000). The appendix brings a very short proof of the proposition, generalizing a technique of Samet (1998).

Proposition 1 Let $\Omega$ be a compact type space with two individuals $i \in\{1,2\}$. Then there is no common prior among them if and only if there is a continuous random variable $f: \Omega \rightarrow R$ for which

$$
\int_{\Omega} f\left(\omega^{\prime}\right) d t_{1}(\omega)<0<\int_{\Omega} f\left(\omega^{\prime}\right) d t_{2}(\omega)
$$

for every $\omega \in \Omega$.

Building on this proposition, we can now provide the positive characterization:

Proposition 2 Let $\Omega$ be a compact type space with two individuals $i \in\{1,2\}$. Then there is no common prior in $\Omega$ if and only if there are expressions $E_{1}, \ldots, E_{n}$ and rational numbers $\left\{p_{k}^{r}, q_{k}^{r}: k=1, \ldots n, \quad r=1, \ldots, s\right\}$ such that whatever is the state of the world $\omega \in \Omega$ which describes the situation at hand, the expression

$$
\bigcup_{r=1}^{s} \bigcap_{k=1}^{n}\left(\neg B_{1}^{p_{k}^{r}} E_{k} \cap B_{2}^{q_{k}^{r}} E_{k}\right)
$$

is common belief at $\omega$, while

$$
\sum_{k=1}^{n} p_{k}^{r}<\sum_{k=1}^{n} q_{k}^{r}
$$

for $r=1, \ldots, s$

Proof. If there is a common prior, there can be no common belief among the individuals that they disagree on the expectation of the random variable $\sum_{k=1}^{n} \chi_{E_{k}}$ (where $\chi_{F}$ is the 
indicator function of the event $F$ ) - see e.g. Cave (1983) or Rubinstein and Wolinsky (1990). But a common belief of such a disagreement follows from (3.2) and (3.3).

In the reverse direction, suppose there is no common prior. By proposition 1, (3.1) holds for some continuous $f$. Since $t_{1}$ and $t_{2}$ are continuous maps, the functions $\mathbf{G}_{i}: \Omega \rightarrow$ $R$ defined by

$$
\mathbf{G}_{i}(\omega)=\int_{\Omega} f\left(\omega^{\prime}\right) d t_{i}(\omega) \quad i=1,2
$$

are continuous. As $\Omega$ is compact, the functions $\mathbf{G}_{i}$ achieve their minimum and maximum on $\Omega$, so for some rational $\varepsilon>0$ we have

$$
\mathbf{G}_{1}(\omega)<-2 \varepsilon<2 \varepsilon<\mathbf{G}_{2}(\omega)
$$

for every $\omega \in \Omega$.

Since $f$ is continuous, for every rational $x$ we have that $f^{-1}(x-\varepsilon, x+\varepsilon)$ is open, and therefore a union of open expressions $\left\{O_{x, \alpha_{x}}\right\} \subseteq \mathcal{O}$. Hence $\cup_{x \in R}\left\{O_{x, \alpha_{x}}\right\}$ is an open cover of $\Omega$, and by the compactness of $\Omega$ it has a finite sub-cover with open expressions $O_{1}, \ldots, O_{m}$, where for $\ell=1, \ldots, m$

$$
O_{\ell} \subseteq f^{-1}\left(x_{\ell}-\varepsilon, x_{\ell}+\varepsilon\right)
$$

for some rational numbers $x_{1}, \ldots, x_{m}$.

Define $F_{1}=O_{1}$, and then inductively $F_{\ell}=O_{\ell} \backslash F_{\ell-1}$ for $k=2, \ldots, m$. Then $\Omega$ is the disjoint union of the expressions $F_{1}, \ldots, F_{m}$. Define

$$
g(\omega)=\sum_{\ell=1}^{m} x_{\ell} \chi_{F_{\ell}}(\omega)
$$

Then $\left|f\left(\omega^{\prime}\right)-g\left(\omega^{\prime}\right)\right|<\varepsilon$ for every $\omega^{\prime} \in \Omega$, and we conclude that

$$
\int_{\Omega} g\left(\omega^{\prime}\right) d t_{1}(\omega)<-\varepsilon<\varepsilon<\int_{\Omega} g\left(\omega^{\prime}\right) d t_{2}(\omega)
$$

for every $\omega \in \Omega$.

Let $d$ the least common denominator of the rational numbers $x_{1}, \ldots, x_{m}$. For some big enough positive integer $c$, the function

$$
h(\omega)=d g(\omega)+c
$$

assumes finitely many positive integer values, and has the form

$$
h(\omega)=\sum_{k=1}^{n} \chi_{E_{k}}(\omega)
$$


where $E_{1}, \ldots, E_{n}$ is the list of expressions in which for $\ell=1, \ldots, m$, and $\omega \in F_{\ell}$, the expression $F_{\ell}$ appears $h(\omega)$ times in the list.

It follows that for $\delta=d \varepsilon>0$

$$
\int_{\Omega} h\left(\omega^{\prime}\right) d t_{1}(\omega)<c-\delta<c+\delta<\int_{\Omega} h\left(\omega^{\prime}\right) d t_{2}(\omega)
$$

for every $\omega \in \Omega$.

Let $L=\left\lceil\frac{n}{\delta}\right\rceil$ and $u_{j}=j \frac{\delta}{n}$ for $j=0, \ldots, L$. Let

$$
p_{k}^{\omega}=\left\{\min _{0 \leq j \leq L} u_{j}: t_{1}(\omega)\left(E_{k}\right)<u_{j}\right\}
$$

and

$$
q_{k}^{\omega}=\left\{\max _{0 \leq j \leq L} u_{j}: t_{2}(\omega)\left(E_{k}\right) \geq u_{j}\right\} .
$$

Then for every $\omega \in \Omega$, the expression

$$
\bigcap_{k=1}^{n}\left(\neg B_{1}^{p_{k}^{\omega}} E_{k} \cap B_{2}^{q_{k}^{\omega}} E_{k}\right)
$$

holds at $\omega$, and by (3.11)

$$
\sum_{k=1}^{n} p_{k}^{\omega}<c<\sum_{k=1}^{n} q_{k}^{\omega}
$$

But the set $\left\{\left(p_{k}^{\omega}, q_{k}^{\omega}\right)_{k=1}^{n}: \omega \in \Omega\right\}$ is finite (the number of its elements does not exceed $\left.(L+1)^{2}\right)$, and therefore it can be written as $\left\{\left(p_{k}^{r}, q_{k}^{r}\right)_{k=1}^{n}: r=1, \ldots s\right\}$ for some finite number $s$. It follows that the expression

$$
\bigcup_{r=1}^{s} \bigcap_{k=1}^{n}\left(\neg B_{1}^{p_{k}^{r}} E_{k} \cap B_{2}^{q_{k}^{r}} E_{k}\right)
$$

holds in all the states of $\Omega$, while

$$
\sum_{k=1}^{n} p_{k}^{r}<\sum_{k=1}^{n} q_{k}^{r}
$$

for $r=1, \ldots, s$. Since $\Omega$ is common belief in any of its states, the conclusion of the proposition follows.

We now turn to generalize this result for more than two individuals. Here again we rely on the following proposition by Feinberg (2000). 
Proposition 3 Let $\Omega$ be a compact type space with individuals $\{1, \ldots, I\}$. Then there is no common prior among them if and only if there are continuous random variables $f_{i}: \Omega \rightarrow R, i=1, \ldots, I$ for which

$$
\sum_{i=1}^{I} f_{i}=0
$$

and

$$
\int_{\Omega} f_{i}\left(\omega^{\prime}\right) d t_{i}(\omega)>0
$$

for every $\omega \in \Omega$.

Proposition 4 Let $\Omega$ be a compact type space with individuals $i \in\{1, \ldots, I\}$. Then there is no common prior among them if and only if there are expressions $\left\{E_{1}^{i}, \ldots, E_{n_{i}}^{i}\right\}_{i=2}^{I}$, a positive integer $c$ and rational numbers $\left\{p_{k, i}^{r}, q_{k, i}^{r}: i=2, \ldots, I, \quad k=1, \ldots n_{i}, \quad r=1, \ldots, s\right\}$ such that whatever is the state of the world $\omega \in \Omega$ which describes the situation at hand, the expression

$$
\bigcup_{r=1}^{s} \bigcap_{i=2}^{I} \bigcap_{k=1}^{n_{i}}\left(\neg B_{1}^{p_{k, i}^{r}} E_{k}^{i} \cap B_{i}^{q_{k, i}^{r}} E_{k}^{i}\right)
$$

is common belief at $\omega$, while

$$
\sum_{k=1}^{n_{i}} q_{k, i}^{r}>c, \quad i=2, \ldots, I
$$

and

$$
\sum_{i=2}^{I} \sum_{k=1}^{n_{i}} p_{k, i}^{r}<(I-1) c
$$

for $r=1, \ldots, s$

Proof. Suppose that (3.20) is common belief while (3.21) and (3.22) obtain. Then there can be no common prior. Indeed, if there had been a common prior $P$, then for

$$
f_{i}=\sum_{k=1}^{n_{i}} \chi_{E_{k}^{i}}-c, \quad i=2, \ldots I
$$


and

$$
f_{1}=-\sum_{i=2}^{I} f_{i}=(I-1) c-\sum_{i=2}^{I} \sum_{k=1}^{n_{i}} \chi_{E_{k}^{i}} .
$$

we would have by (3.21) and (3.22) that ${ }^{6}$ for every $\omega \in \Omega$

$$
\int_{\Omega} f_{i} \mathrm{~d} t_{i}(\omega)>0, \quad i=1, \ldots I
$$

and therefore

$$
\int_{\Omega} f_{i} \mathrm{~d} P>0, \quad i=1, \ldots I
$$

which is impossible since $\sum_{i=1}^{I} f_{i}=0$.

In the reverse direction, suppose there is no common prior. By proposition 3 there are continuous random variables $f_{i}: \Omega \rightarrow R, i=1, \ldots, I$ for which (3.18) and (3.19) obtain. Since $t_{i}$ are continuous maps, the functions $\mathbf{G}_{i}: \Omega \rightarrow R$ defined by

$$
\mathbf{G}_{i}(\omega)=\int_{\Omega} f_{i}\left(\omega^{\prime}\right) d t_{i}(\omega) \quad i=1, \ldots I
$$

are continuous. As $\Omega$ is compact, the functions $\mathbf{G}_{i}$ achieve their minimum and maximum on $\Omega$, so for some rational $\varepsilon>0$ we have

$$
\mathbf{G}_{i}(\omega)>I \varepsilon \quad i=2, \ldots I
$$

for every $\omega \in \Omega$.

Since the functions $f_{i}$ are continuous, for every rational $x$ we have that $f_{i}^{-1}(x-\varepsilon, x+\varepsilon)$ is open, and therefore a union of open expressions $\left\{O_{x, \alpha_{x}^{i}}^{i}\right\} \subseteq \mathcal{O}$. Hence $\cup_{x \in R}\left\{O_{x, \alpha_{x}^{i}}^{i}\right\}$ is an open cover of $\Omega$, and by the compactness of $\Omega$ it has a finite sub-cover with open expressions $O_{1}^{i}, \ldots, O_{m_{i}}^{i}$, where for $\ell=1, \ldots, m_{i}$

$$
O_{\ell}^{i} \subseteq f_{i}^{-1}\left(x_{\ell}^{i}-\varepsilon, x_{\ell}^{i}+\varepsilon\right)
$$

for some rational numbers $x_{1}^{i}, \ldots, x_{m_{i}}^{i}$.

Define $F_{1}^{i}=O_{1}^{i}$, and then inductively $F_{\ell}^{i}=O_{\ell}^{i} \backslash F_{\ell-1}^{i}$ for $k=2, \ldots, m_{i}$. Then for every $i=1, \ldots, I$, the space $\Omega$ is the disjoint union of the expressions $F_{1}^{i}, \ldots, F_{m_{i}}^{i}$. Define

$$
g_{i}(\omega)=\sum_{\ell=1}^{m_{i}} x_{\ell}^{i} \chi_{F_{\ell}^{i}}(\omega)
$$

\footnotetext{
${ }^{6}$ Recall that we assume $\Omega$ does not contain proper common-belief sub-components.
} 
Then

$$
\left|g_{i}\left(\omega^{\prime}\right)-f_{i}\left(\omega^{\prime}\right)\right|<\varepsilon
$$

and

$$
\left|-\sum_{i=2}^{I} g_{i}\left(\omega^{\prime}\right)-f_{1}\left(\omega^{\prime}\right)\right|=\left|-\sum_{i=2}^{I} g_{i}\left(\omega^{\prime}\right)+\sum_{i=2}^{I} f_{i}\left(\omega^{\prime}\right)\right|<(I-1) \varepsilon
$$

for every $\omega^{\prime} \in \Omega$, so we conclude from (3.28) and (3.31) that

$$
\int_{\Omega} g_{i}\left(\omega^{\prime}\right) d t_{i}(\omega)>(I-1) \varepsilon \geq \varepsilon, \quad i=2, \ldots I
$$

and from (3.28) and (3.32) that

$$
-\int_{\Omega} \sum_{i=2}^{I} g_{i}\left(\omega^{\prime}\right) d t_{1}(\omega)>\varepsilon
$$

for every $\omega \in \Omega$.

Let $d$ the least common denominator of the rational numbers $\left\{x_{1}^{i}, \ldots, x_{m_{i}}^{i}\right\}_{i=2}^{I}$. For some big enough positive integer $c$, the functions

$$
h_{i}(\omega)=d g_{i}(\omega)+c
$$

assume each finitely many positive integer values, and have the form

$$
h_{i}(\omega)=\sum_{k=1}^{n_{i}} \chi_{E_{k}^{i}}(\omega)
$$

where $E_{1}^{i}, \ldots, E_{n_{i}}^{i}$ is the list of expressions in which for $\ell=1, \ldots, m_{i}$, and $\omega \in F_{\ell}^{i}$, the expression $F_{\ell}^{i}$ appears $h_{i}(\omega)$ times in the list.

It follows from (3.33) and (3.34) that for $\delta=d \varepsilon>0$

$$
\begin{aligned}
\int_{\Omega} h_{i}\left(\omega^{\prime}\right) d t_{i}(\omega) & >c+\delta, \quad i=2, \ldots I \\
\int_{\Omega} \sum_{i=2}^{I} h_{i}\left(\omega^{\prime}\right) d t_{1}(\omega) & <(I-1)(c+\delta)
\end{aligned}
$$

for every $\omega \in \Omega$.

$$
\begin{gathered}
\text { Let } n=\max _{2 \leq i \leq I} n_{i}, L=\left\lceil\frac{n}{\delta}\right\rceil \text { and } u_{j}=j \frac{\delta}{n} \text { for } j=0, \ldots, L \text {. Let } \\
p_{k, i}^{\omega}=\left\{\min _{0 \leq j \leq L} u_{j}: t_{1}(\omega)\left(E_{k}^{i}\right)<u_{j}\right\}
\end{gathered}
$$


and

$$
q_{k, i}^{\omega}=\left\{\max _{0 \leq j \leq L} u_{j}: t_{i}(\omega)\left(E_{k}^{i}\right) \geq u_{j}\right\}, \quad i=2, \ldots I .
$$

Then for every $\omega \in \Omega$, the expression

$$
\bigcap_{i=2}^{I} \bigcap_{k=1}^{n_{i}}\left(\neg B_{1}^{p_{k, i}^{\omega}} E_{k}^{i} \cap B_{i}^{q_{k, i}^{\omega}} E_{k}^{i}\right)
$$

holds at $\omega$, and by (3.37) and (3.38)

$$
\begin{aligned}
\sum_{k=1}^{n_{i}} q_{k, i}^{\omega} & >c \\
\sum_{i=2}^{I} \sum_{k=1}^{n_{i}} p_{k, i}^{\omega} & <(I-1) c
\end{aligned}
$$

But the set $\left\{p_{k, i}^{\omega}, q_{k, i}^{\omega}: i=2, \ldots, I, \quad k=1, \ldots n_{i}, \quad \omega \in \Omega\right\}$ is finite (the number of its elements does not exceed $\left.(L+1)^{2 I}\right)$, and therefore it can be written as $\left\{p_{k, i}^{r}, q_{k, i}^{r}: i=2, \ldots, I, \quad k=1, \ldots n_{i}, \quad r=1, \ldots, s\right\}$ for some finite number $s$. It follows that the expression

$$
\bigcup_{r=1}^{s} \bigcap_{i=2}^{I} \bigcap_{k=1}^{n_{i}}\left(\neg B_{1}^{p_{k, i}^{r}} E_{k}^{i} \cap B_{i}^{q_{k, i}^{r}} E_{k}^{i}\right)
$$

holds in all the states of $\Omega$, while

$$
\begin{aligned}
\sum_{k=1}^{n_{i}} q_{k, i}^{r} & >c \\
\sum_{i=2}^{I} \sum_{k=1}^{n_{i}} p_{k, i}^{r} & <(I-1) c
\end{aligned}
$$

for $r=1, \ldots, s$. Since $\Omega$ is common belief in any of its states, the conclusion of the proposition follows. 


\section{Appendix}

A short proof of Proposition 1. For each of the players $i=1,2$, the map $\omega \rightarrow$ $t_{i}(\omega)$ is continuous. Hence the image of this map, $t_{i}(\Omega)$ - player $i$ 's set of types - is a compact subset of $\Delta(\Omega)$, and therefore so is its closed convex hull - player $i$ 's set of priors $\mathcal{P}\left(t_{i}(\Omega)\right){ }^{7}$ Thus, when there is no common prior, the two sets of priors can be strongly separated: There is a continuous linear functional $F$ on $\Delta(\Omega)$ and a number $c$ for which $F\left(p_{1}\right)<c<F\left(p_{2}\right)$ for priors $p_{1}, p_{2}$ of players 1 and 2 (and in particular their types $\left.t_{1}(\omega), t_{2}(\omega)\right)$, respectively.

By the Riesz representation theorem, $\Delta(\Omega)$ is the space of continuous linear functionals on the space $C(\Omega)$ of continuous functions on $\Omega$, with the definition $\mu(f)=$ $\int_{\Omega} f(\omega) \mathrm{d} \mu(\omega)$ for $f \in C(\Omega)$ and $\mu \in \Delta(\Omega)$. Furthermore, the real valued mapping $\langle\mu, f\rangle \equiv \int_{\Omega} f(\omega) \mathrm{d} \mu(\omega)$ from $\Delta(\Omega) \times C(\Omega)$ is bilinear and separates the points of $\Delta(\Omega)$ and $C(\Omega)$. Hence, when $\Delta(\Omega)$ is endowed with the weak-* topology, $C(\Omega)$ is the space of continuous linear functionals on $\Delta(\Omega)$, with the definition $f(\mu)=\int_{\Omega} f(\omega) d \mu(\omega)$ (see e.g. Aliprantis and Border (1999, p. 208, thm. 5.83) ${ }^{8}$

Therefore, when there is no common prior, there is a continuous function $g \in C(\Omega)$ such that $\int_{\Omega} g(\omega) \mathrm{d} t_{1}(\omega)<c<\int_{\Omega} g(\omega) \mathrm{d} t_{2}(\omega)$ for every $\omega \in \Omega$. Defining $f=g-c$, we get $\int_{\Omega} f(\omega) \mathrm{d} t_{1}(\omega)<0<\int_{\Omega} f(\omega) \mathrm{d} t_{2}(\omega)$, so $f$ is a zero-sum bet that both players like to take.

This proof generalizes a proof of Samet (1998). Samet's proof of the characterization of common priors for more than two players can now be generalized using the same technique to yield a short proof of proposition 3 .

${ }^{7}$ More explicitly, the mapping $\mathcal{P}: \Delta(\Omega) \rightarrow \Delta(\Omega)$ defined by

$$
\int_{\Omega} f(\omega) \mathrm{d}(\mathcal{P}(\nu))(\omega)=\int_{\Omega}\left(\int_{\Omega} f\left(\omega^{\prime}\right) \mathrm{d}\left(t_{i}(\omega)\left(\omega^{\prime}\right)\right) \mathrm{d} \nu(\omega)\right.
$$

for real valued continuous functions $f \in C(\Omega)$ is continuous, because the integrand on the right-hand side is a real-valued continuous function on $\Delta(\Omega)$ (if $\left\{\nu_{\alpha}\right\}$ is a net in $\Delta(\Omega)$ that converges to $\nu \in \Delta(\Omega)$, then

$$
\int_{\Omega}\left(\int _ { \Omega } f ( \omega ^ { \prime } ) \mathrm { d } ( t _ { i } ( \omega ) ( \omega ^ { \prime } ) ) \mathrm { d } \nu _ { \alpha } ( \omega ) \rightarrow \int _ { \Omega } \left(\int_{\Omega} f\left(\omega^{\prime}\right) \mathrm{d}\left(t_{i}(\omega)\left(\omega^{\prime}\right)\right) \mathrm{d} \nu(\omega)\right.\right.
$$

for every $f \in C(\Omega)$, since $\int_{\Omega} f\left(\omega^{\prime}\right) \mathrm{d}\left(t_{i}(\omega)\left(\omega^{\prime}\right)\right.$ is continuous on $\Omega$ for each such $f$, and hence

$$
\int_{\Omega} f(\omega) \mathrm{d}\left(\mathcal{P}\left(\nu_{\alpha}\right)\right)(\omega) \rightarrow \int_{\Omega} f(\omega) \mathrm{d}(\mathcal{P}(\nu))(\omega)
$$

for every $f \in C(\Omega)$, which, by definition of the weak-* topology on $\Delta(\Omega)$, implies that $\mathcal{P}\left(\nu_{\alpha}\right) \rightarrow \mathcal{P}(\nu)$ ). Therefore $\mathcal{P}(\Delta(\Omega))$ - the set of priors of player $i$ - is compact as the continuous image of a compact set.

${ }^{8} \mathrm{I}$ 'm grateful to Kim Border for pointing out to me this property. 


\section{$5 \quad$ References}

Aliprantis, R. and K. Border (1999), Infinite Dimensional Analysis - a Hitchhiker's Guide, 2nd Edition, Springer

Billingsley, P. (1968), Convergence of Probability measures, John Wiley \& Sons

Cave, J.A.K. (1983), Learning to Agree, Economics Letters 12, 147-152

Feinberg, Y. (2000), Characterizing Common Priors in the Form of Posteriors, Journal of Economic Theory 91, 127-179

Halpern, J.Y. (1998), Characterizing the Common Prior Assumption, in "Theoretical Aspects of Rationality and Knowledge" (I. Gilboa, ed.), Proceedings of the 7th conference, 133-146, Morgan Kaufman.

Harsanyi, J.C. (1967-68), Games with Incomplete Information Played by Bayesian Players, parts I-III, Management Science, 14, 159-182, 320-334, 486-502.

Mertens, J.F. and S. Zamir (1985), Formulation of Bayesian Analysis for Games with Incomplete Information, International Journal of Game Theory 14, 1-29.

Morris, S. (1994), Trade with heterogeneous prior beliefs and asymmetric information, Econometrica 62, 1327-1347

Morris, S. (1995), The Common Prior Assumption in Economic Theory, Economics and Philosophy 11, 227-253

Rubinstein, A. and A. Wolinsky (1990), On the Logic of "Agreeing to Disagree" Type Results, Journal of Economic Theory 51, 184-193

Samet, D. (1998a), Common Priors and the Separation of Convex Sets, Games and economic Behavior 24, 172-174

Samet, D. (1998b), Iterated Expectations and Common Priors, Games and economic Behavior 24, 131-141 